\title{
Smart UNL: un Concepto de Universidad Inteligente
}

\section{Smart UNL: A Smart University Concept}

Andy Vega ${ }^{1}$ https://orcid.org/0000-0003-0106-6880

Kleber Morillo 1 https://orcid.org/0000-0002-2839-3104

Christian Campoverde ${ }^{1}$ https://orcid.org/0000-0002-1686-8299

Luis Rodríguez 1 https:///orcid.org/0000-0003-3134-7157

${ }^{1}$ Universidad Nacional de Loja, Loja, Ecuador

andy.vega@unl.edu.ec

kleber.morillo@unl.edu.ec

christian.campoverdedunl.edu.ec

luis.e.rodriguez@unl.edu.ec

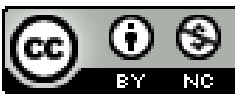

Esta obra está bajo una licencia internacional

Creative Commons Atribución-NoComercial 4.0.
Enviado:

Aceptado:

Publicado:
$2021 / 03 / 05$

$2021 / 06 / 15$

$2021 / 06 / 30$

\section{Resumen}

Se exponen los argumentos para pensar en la evolución de la educación superior en consonancia con el desarrollo tecnológico, y se contextualiza el concepto de Modelo de Universidad Inteligente. El propósito de este artículo es presentar acciones que estructuren un entorno universitario inteligente para la Universidad Nacional de Loja (UNL), en el marco de sus ejes estratégicos, que definen y caracterizan el proyecto Smart UNL. Luego se propone una ruta que engloba los ámbitos de la gestión, administración, academia e investigación universitaria a partir de los ejes de gobierno y condiciones tecnológicas de la institución, con la finalidad de que la universidad pueda transitar hacia los sistemas inteligentes. Finalmente, se articula el modelo de universidad inteligente Smart UNL a una propuesta de arquitectura tecnológica para la gestión de información, con un sistema de datos abiertos que posibilite la investigación y el desarrollo de nuevos proyectos.

Palabras clave: universidad, inteligente, arquitectura, smart.

\begin{abstract}
The evolutionary arguments of higher education in the face of technology are evidenced and the concept of the Smart University Model is contextualized. The purpose of this article is to present actions that structure an intelligent university environment within the National University of Loja (UNL), evidencing its strategic axes that define and characterize the Smart UNL project developed in this academic entity. Then a route is proposed that encompasses the areas of university management, administration, academia and research based on the governance axes and technological conditions of the institution, so that the university can move on the way to intelligent systems. Finally, the UNL smart
\end{abstract}

Sumario: 1 Introducción, 2 Materiales y Métodos, 3 Resultados, 4 Conclusiones.

Como citar: Vega, A., Morillo, K., Campoverde, C., \& Rodríguez, L. (2021). Smart UNL: un Concepto de Universidad Inteligente. Revista Tecnológica - Espol, 33(1), 137-149.

http://www.rte.espol.edu.ec/index.php/tecnologica/article/view/813 
university model is articulated to a technological architecture proposal for information management using open data for research and development of new projects.

Keywords: University, smart, architecture, technology.

\section{Introducción}

La Universidad Nacional de Loja, con 160 años de historia, es la segunda institución de educación superior más antigua del Ecuador; ubicada en la región sur del país, actualmente, acoge a más de 10000 estudiantes, distribuidos en cinco facultades. El desarrollo inevitable de las Tecnologías de la Información y la Comunicación (TIC) ha marcado un antes y un después en el cotidiano social; esto pese a que el estilo de vida diario de las personas en el Ecuador aún no se encuentra afectado de modo considerable por la presencia de la tecnología, si se compra con otros países desarrollados. Esto se evidencia, por ejemplo, en la ubicación que ocupa el Ecuador en el Índice Global de Innovación, elaborado por la compañía Bloomberg, y que establece una clasificación de los países más innovadores del mundo; dicho índice valora cuestiones como el registro de patentes, la inversión en educación o la productividad; son diez países que lideran este ranking, y nuestro país no aparece ni de cerca en los 50 primeros lugares (Jamrisko y Lu, 2020), lo que indica que aún los hábitos de vida, trabajo, movilidad, salud y educación en general aún no están integrados al desarrollo tecnológico. Como entidades universitarias, las aplicaciones digitales y los nuevos modos de flujo de información estimulan a repensar y buscar nuevos procesos de cambio y adaptación para impulsar el desarrollo social. En un campus universitario confluyen cientos de personas todos los días con diferentes requerimientos, por lo tanto, toda institución debe estar lista para brindar variados servicios a sus usuarios: matriculación de estudiantes; trámites académicos para docentes e investigadores; gestión de quintas; manejo de desperdicios; mantenimiento eléctrico e infraestructura, etc. Entonces, ¿por qué no trabajar para convertir dicho espacio en un campus inteligente, entendiendo por este término todo lo que engloba el concepto Smart? Para lograr dicha evolución, la UNL deberá propiciar cambios significativos en diversos campos, como, por ejemplo, en la actualización profesional del talento humano para alcanzar el desarrollo y la calidad en el servicio; además, se deberán implementar modelos de gestión modernos que incorporen formas de relación y participación entre los distintos actores implicados (alumnos, docentes y administrativos). Esto supondría para la institución contar con un nuevo paradigma de administración, control y gestión, enmarcado en los conceptos de Smart University. Para alcanzar un nuevo modelo de educación superior no solo se requeriría afrontar amplios desafíos y retos innumerables, sino que también se deberá mejorar la eficiencia de la Universidad bajo parámetros innovadores y de alta creatividad. Además, esto facilitaría a la institución incorporar niveles de competitividad que le permitan situarse en la vanguardia educativa. Por otro lado, es necesario incorporar nuevos procesos de formación, capaces de articular las nuevas exigencias sociales, culturales y educativas en consonancia con las nuevas tecnologías y el cuidado del medio ambiente. El presente trabajo ofrece una propuesta de acciones enmarcadas en el concepto Smart Campus que posibiliten a la Universidad Nacional de Loja incorporar sistemas inteligentes en la gestión administrativa, académica e investigativa; para ello, como primer paso, se indagó y evaluó experiencias de instituciones de educación superior que han desarrollado esta propuesta en los últimos años. Esta investigación previa permitió identificar como un elemento principal la oferta de servicios altamente tecnologizados -que se apoyen en el entorno y la necesidad institucional de acuerdo con las especificidades del campus e, incluso, de otros escenarios-, que permitan una mejor organización de los procesos internos y se traduzcan en una operación académica eficiente. Finalmente, incorporar una infraestructura de espacios y aulas inteligentes e innovadoras facilitará la relación entre administrativos, docentes y alumnos, a partir del desarrollo de hardware y software que operen de forma autónoma.

\section{Materiales y Métodos}

Para llevar a cabo el presente trabajo se investigó el escenario de la educación superior en términos generales, analizando su proceso evolutivo, destacando los momentos significativos en los que las Tecnologías de Información y Comunicación (TIC) han formado parte fundamental de su desarrollo. Posteriormente se efectuó una revisión bibliográfica de la mayor cantidad de literatura internacional (empresarial y académica) acerca de las Universidades Inteligentes o Smart University 
para conocer sus conceptos, los parámetros que las definen y los criterios para evaluarlas. Con la ayuda de las experiencias recabadas de otros contextos académicos, en los cuales los conceptos y caminos hacia una universidad inteligente ya han sido abordados, se propusieron los ejes estructurantes y estratégicos que definen y caracterizan al proyecto Smart UNL. Luego, fue necesario analizar los elementos de hardware informático y de telecomunicaciones de la institución, como paso fundamental para desarrollar el concepto de Smart University. En esta fase, se realizó una identificación y revisión de estos recursos con el objetivo de articularlos con los ejes tecnológicos identificados. Finalmente, se vinculó una propuesta de arquitectura inteligente para gestión de la información a partir del uso de datos abiertos que posibiliten la investigación y el desarrollo de proyectos, de tal forma que funcionen de manera sinérgica en la construcción de nuevos sistemas para gestión, administración, desarrollo académico e investigativo dentro de la universidad.

\section{La Evolución de las Tecnologías en la Educación Superior}

El vertiginoso desarrollo de las TIC, iniciado a finales del siglo XX, influye de manera transversal en un amplio e incontable grupo de actividades sociales: la industria, el transporte, la salud y, sin duda, la educación. Esta evolución genera un nuevo paradigma de desarrollo en varias áreas disciplinares, por ejemplo, en el de la educación superior, en donde adquiere un protagonismo inédito respecto a sus estrategias de formación.

Por otra parte, la incursión de nuevas formas de economía, como la "economía en internet o web", genera intensas transformaciones en los procesos de educación superior, lo que ha obligado a esta adoptar novedosas estrategias de gestión institucional y oferta académica variada y actualizada, todo en el marco de una formación académica de calidad.

De acuerdo con la Conferencia Mundial sobre la Educación Superior, llevada a cabo en el año 2009, los estudiantes deben asumir la responsabilidad de ser un participante activo en la apropiación del conocimiento, los valores y las habilidades necesarios para aprender a conocer, hacer, trabajar en equipo, así como a ser solidario, tomar decisiones, resolver problemas, etc. (Unesco, 1989). Asimismo, se recomienda crear nuevos entornos pedagógicos que incluyan servicios de educación a distancia, plataformas y sistemas virtuales de enseñanza superior, capaces de ofrecer una educación de alta calidad que contribuya al progreso social y económico, así como a la sostenibilidad y a la cobertura de las necesidades sociales más importantes (IESALAC, 1998). Además, la figura del maestro será ahora la del facilitador del aprendizaje, quien deberá ser percibido por los alumnos como un amigo, como alguien que los escucha y ayuda a desarrollarse para que adquieran destrezas y habilidades. (Unesco, 1998).

De acuerdo con Sangrá, A. (2001) son diferentes las respuestas que las universidades han dado a esta nueva realidad. Por ejemplo, en el año 2001 se clasificó y diferenció a la universidad según su grado de virtualidad, a partir de los siguientes criterios:

a) Universidad virtual: concepto global e integral que suponen una realidad educativa universitaria totalmente virtual, tanto para los alumnos como para el personal docente e investigador.

b) Campus virtual: el campus virtual es una metáfora de enseñanza, aprendizaje e investigación creado por la convergencia de las poderosas nuevas tecnologías de la información y la comunicación.

c) Cursos on-line: se encuentran en un tercer estadio de concreción; se trata de una oferta directa de contenido, sin pretender establecer una relación de pertenencia con la institución que los ofrece.

d) E-learning: puede definirse como "el uso de tecnologías basadas en internet, para proporcionar un amplio abanico de soluciones que aúnen adquisición de conocimiento y habilidades o capacidades". 
Además, se establecen diferentes criterios para alcanzar el sistema e-learning: 1) Que se realice en red; 2) Que se haga llegar al usuario final a través de un computador que utilice estándares tecnológicos de internet; 3) Que esté centrado en la más amplia visión de soluciones a los problemas de aprendizaje, que implica superar los paradigmas tradicionales de la formación.

Para la primera década del nuevo milenio, también aparecieron otras propuestas atractivas hacia la evolución de las tecnologías en la educación superior y que plantean un enfoque diferente a las antes mencionadas. Estos nuevos modelos bosquejan una propuesta de clasificación que tiene como criterio fundamental la complejidad y madurez de la incorporación de las TIC (Senescyt, 2018):

- Modelo de iniciación. Nivel básico que se caracteriza por ofrecer los apuntes de la clase presencial, mínimamente transformados, en la red. Generalmente no se ofrecen oportunidades para la interacción. Trata de utilizar las TIC e internet como apoyo en el aprendizaje y en la enseñanza, pero sin que se produzca un cambio de cultura ni de profesores ni de alumnos.

- Modelo estándar. Trata de utilizar activamente las ventajas ofrecidas por la tecnología para permitir un cierto grado de comunicación e interacción. Recursos electrónicos, tareas, guías, etc.

- Modelo evolucionado, introduce elementos complementarios para mejorar tanto el entorno como el aprendizaje: animaciones, clases pregrabadas y en vivo, etc.

- Modelo radical. Los tres anteriores tratan de adaptar el modelo de enseñanza tradicional, este, en cambio ignora el concepto de clases.

Esta breve descripción evidencia que el uso de las TIC en las universidades está aportando significativamente al proceso de enseñanza-aprendizaje, tanto en espacios regionales como nacionales o internacionales. Por ejemplo, el vertiginoso crecimiento de la Web 2.0 y 3.0 crea un nuevo escenario con distintas y ampliadas formas de aprender y de concebir el conocimiento, y, por lo tanto, de gestionar las instituciones educativas. Actualmente, la educación superior en el mundo se encuentra viviendo momentos inéditos provocados por los avances tecnológicos: matrículas $100 \%$ en línea, trámites cero papeles y robustas plataformas de sistemas de gestión académica y administrativa, entre otros. Esto marca una diferencia importante con respecto a la inmovilidad que por décadas caracterizó a las universidades. Por otro lado, actualmente es necesario identificar las condiciones que impulsen el progreso y desarrollo de estas. La tecnología, el acceso a la información y el proceso de globalización deben aportar a este nuevo momento de la universidad. En el Ecuador existe un vertiginoso crecimiento de profesionales especialistas en tecnologías, lo que facilitaría transitar hacia ese nuevo escenario (Senescyt, 2018). A partir de las novedosas herramientas científicas que se han desarrollado en las últimas décadas, es posible buscar y experimentar nuevos procesos de acción dentro de las universidades, que influyan notablemente a un cambio de gestión, administración y educación. En este sentido, por ejemplo, la tecnología facilita el acceso abierto y permanente de contenidos, datos e información al estudiante, lo que garantiza un pertinente proceso de formación académica. Asimismo, la tecnología permite al docente acceder a proyectos internacionales de investigación que posibilitan el contacto con colegas de diferentes países e interesantes intercambios académicos. Esto genera una dinámica en red a partir de los flujos de contactos y proyectos que emergen a escala regional y global, dando mayor visibilidad y difusión a la institución y retroalimentando la producción de conocimiento.

La tecnología, el acceso a la información junto con la globalización constituyen los tres pilares que posibilitan la evolución del modelo universitario. Esto se evidencia, por ejemplo, en el crecimiento de la demanda en América latina y el Caribe (ALC) de los servicios educativos universitarios: el porcentaje de individuos entre los 18 y 24 años inscritos en la educación superior en ALC creció del 21 $\%$, en 2000, al $40 \%$, en 2010. Esto pese a que la desigualdad en el acceso aún es notoria en esta región, aunque, por otro lado, se registran avances considerables en este rubro, en particular, entre grupos de ingreso bajo y medio. En 2000, el $50 \%$ más pobre de la población representaba el $16 \%$ de los alumnos de educación superior; en 2013, esta cifra creció al $25 \%$, aproximadamente. En términos generales, durante los últimos veinte años, la matrícula de educación superior en el mundo casi se duplicó, de 19 
$\%$ al $38 \%$, entre 2000 y 2018. Sin embargo, sigue habiendo grandes diferencias entre las regiones (Ferreyra, Avitabile, Botero, Haimovich y Urzúa, 2017).

Sin duda, el escenario de evolución de la educación superior ha sido favorable en los últimos años, incluso, pese a que después del primer trimestre del año 2020 surgió la pandemia generada por el virus SARS-CoV-2 (COVID-19). El presente año de pandemia ha originado un impacto profundo en el desarrollo de la educación. Las universidades permanecen aún cerradas, y, en los momentos más críticos, se estima que 220 millones de estudiantes de educación superior se vieron afectados por los cierres de los centros educativos en todo el mundo, de los cuales 60 millones pertenecen a Latinoamérica. Además, cabe advertir que un porcentaje alto de ese $60 \%$ corresponde a estudiantes provenientes de sectores pobres, que deben lidiar dentro de contextos socioeconómicos precarios y con sistemas de educación a distancia, en buena medida, improvisados. Las pérdidas educativas, probablemente, sean importantes, al igual que es posible que se agudicen las desigualdades y las deserciones dentro del sistema educativo. En este contexto, la educación superior en América Latina y el mundo deberá tomar un rol protagónico y relevante en los procesos de transformación social. Sin duda, se volverá esencial la capacidad de adaptación que tengan las universidades frente a las difíciles circunstancias presentes u otras similares que se puedan presentar en el futuro. Sobre todo, esta capacidad de adaptación tendrá especial importancia en ámbitos como los de la gestión institucional, administrativa, formación académica e investigativa, pues será fundamental ganar en eficiencia, competitividad, inclusión y calidad educativa, en un marco de apertura, flexibilidad, creatividad e innovación permanente (Centros de Estudio de la Educación Argentina, 2021).

\section{La Universidad Inteligente, una comunidad que aprende}

La presencia de las tecnologías electrónicas y el avance de las TIC en los sistemas educativos abren un abanico de posibilidades para promover nuevas estrategias de gestión eficiente dentro de las universidades. Po lo tanto, la necesidad de romper viejos paradigmas de educación e implementar nuevas herramientas de trabajo con características dinámicas, eficientes y tecnologías, en donde se evidencien procesos innovadores, es prioritario. El concepto "Smart Cities" o "Ciudades Inteligentes" gira en torno a mejorar la calidad de vida de las personas a partir de incorporar procesos eficientes en su cotidianidad, sin que el medioambiente se vea afectado. En este escenario, la automatización y el desarrollo tecnológico juegan un rol fundamental para tales fines. Según Tkchuck (2012), el concepto de Universidad Inteligente o Smart University se define como un "modelo organizacional que da respuesta a las nuevas necesidades de las instituciones educativas frente a un contexto de sociedad digital que se enmarca en la Era de la Información y el Conocimiento".

La Universidad Inteligente deberá evidenciar un entorno académico cuya visión y operaciones se traduzcan en un campo integral y sistémico, donde cada actor, espacio o área se entrelacen de forma transparente en un trabajo sinérgico y coordinado a través del uso de tecnologías electrónicas, sistemas digitales, infraestructura de comunicación, software especializado, personal capacitado y calificado, con el único afán de establecer una gestión altamente eficiente.

El desarrollo de un modelo de Universidad Inteligente se refleja a partir de la creación de un ecosistema académico que da lugar a una institución universitaria integrada, basada en los siguientes factores:

- La innovación como eje de dinámicas operativas abiertas e interconectadas

- La aplicación de inteligencia colectiva con efectos multidisciplinares

- La universidad como nodo de un sistema integral, evolutivo, dinámico y acumulativo

- Mecanismos de coordinación y equilibrio entre tecnología y medioambiente en la vida universitaria

- Las TIC como herramientas principales que convalidan una gestión inteligente de la universidad (Tkchuck, 2012) 
La inteligencia colectiva y su aprendizaje se basan en la interacción entre pares, y constituye una condición sine qua non que toda institución deberá aprender y poner en práctica para alcanzar niveles de gestión eficientes y trabajo en equipo, anteponiendo la ayuda recíproca a los intereses de reconocimiento personales. Con el fin de comprender los desafíos que tiene por delante el proceso de enseñanza-aprendizaje bajo este nuevo ecosistema académico, es oportuno distinguir las características de tres actores clave: alumnos, profesores y universidad. Los alumnos universitarios actuales se perciben a sí mismos multimediáticos, comprometidos y como miembros de comunidades digitales, así como con la capacidad de aprender dentro de un entorno académico digital compatible con las necesidades y formas de desempeño profesional futuro. En cuanto a los profesores, la tendencia es más diversa, pues se presentan entre ellos perfiles diferentes con respecto a su función docente. Para algunos, la experticia profesional supone ser la fuente primaria de conocimientos y experiencias para transmitir en el aula, en tanto que para otros la labor investigativa es el centro de sus estrategias de enseñanza, como generadores e impulsores de conocimientos. En ambos casos, puede concebirse a los docentes como facilitadores y transmisores estratégicos de conocimiento e información en la era de la información actual; este rol cobra mayor relevancia en relación con las prácticas de incorporación de contenidos específicos (Tkchuck, 2012). Por último, la universidad adquiere mayor protagonismo en el proceso de adquisición de conocimientos, pero de manera cada vez más coordinada con el resto de las instituciones y organizaciones educativas, dando lugar a una dinámica desde donde se generan retroalimentaciones y sinergias a partir de redes de conocimiento e intercambio.

Las nuevas formas de socialización exigen cambios en el ámbito del conocimiento y el aprendizaje desde el momento en que impactan sobre los paradigmas metodológicos. De alguna forma, el saber deja de ser un cuerpo estático de acceso único y rígido para dar paso a la apertura de nuevos canales de información, la experiencia y el conocimiento, lo que evidencia un marco de innovación disruptiva, de inteligencia colectiva, design thinking (pensamiento de diseño), de desarrollo de nuevas soluciones y formas de investigación (interconectada y en red) que incorporan la creatividad tecnológica con un enfoque social, ambiental y cultural. En otras palabras, estas nuevas formas de socialización, que expresan la dinámica propia de una Universidad Inteligente, demandan un ecosistema académico holístico e innovador que impacte transversalmente en todas las áreas institucionales de la universidad e involucre a cada uno de sus actores (Tkchuck, 2012).

Es importante destacar que la idea de "inteligencia" en las instituciones y, concretamente, en las universidades se vincula con el desarrollo de un entorno capaz de crear redes de cooperación e intercambio, así como de promover ideas fecundas que propicien un entorno capaz de valorar capacidades y esfuerzos de cada uno de los actores comprometidos con una visión innovadora de universidad. Bajo este modelo, aun cuando las TIC poseen especial protagonismo, no debemos dejar de considerar que constituyen sólo un medio que permite desarrollar dinámicas de sinergia dentro de la comunidad universitaria y otorgar el sustento necesario para los ejes y características que definen una Universidad Inteligente.

El concepto de Universidad Inteligente se funda sobre la idea de que las universidades son "organizaciones que aprenden", ya que se conciben a sí mismas como espacios flexibles y creativos, con capacidad de adaptación a nuevos escenarios. En este sentido, el aprendizaje organizativo se vuelve el instrumento esencial que garantiza la supervivencia de cualquier organización en general y de las universidades en particular en el siglo XXI.

Cuando las transformaciones penetran el orden social conocido hasta entonces, se impone la necesidad de contar con un corpus específico de saberes y prácticas que convaliden la creación de un nuevo orden organizacional (Tkchuck, 2012).

\section{Ejes estructurantes para un entorno universitario inteligente}

1) Economía: consiste en redes inteligentes de trabajo e intercambio, desmaterialización de documentos, gestiones digitales, teletrabajo. Se vincula con una cultura que impulse el espíritu emprendedor profesional y personal. 
2) Movilidad: se caracteriza por reestructurar las soluciones logísticas implicadas en el sistema universitario (tanto presencial como a distancia), incentivar el uso de la movilidad sustentable (bicicleta), brindar un servicio de transporte sustentable.

3) Gobierno: se caracteriza por mantener una política de datos abiertos (mayor transparencia), sistemas de seguridad inteligentes, ciudadanía universitaria digital (inteligente). Se vincula con la idea de que la toma de decisiones sea participativa y abierta mediante una gestión institucional transparente; la digitalización y modernización de los procesos de gestión son la condición para mejorar el vínculo entre la institución y la comunidad universitaria a través de dinámicas de acceso a la información.

4) Calidad de Vida: métricas inteligentes de energía, servicios específicos de salud y asistencia universitaria. Apunta a mejorar la calidad de vida a través de la integración cultural de los miembros de la comunidad universitaria.

5) Medio ambiente sustentable: las nuevas tecnologías pueden utilizarse para proteger y preservar los recursos naturales, reduciendo la contaminación y mejorando la protección ambiental. De ahí que se vuelve imprescindible alentar políticas que impulsen el cuidado del medio ambiente, así como incorporar innovaciones sustentables en la infraestructura universitaria: iluminación inteligente; (LED), gestión del agua y sistema de riego inteligentes; política de "basura cero"; uso de energías limpias y renovables, a fin de mejorar la eficiencia energética de la institución. Asimismo, será necesario promover el uso adecuado de los recursos naturales, las prácticas de reciclaje y de selección de residuos (orgánicos e inorgánicos).

6) Ciudadanía: educación e investigación a fin de contar con una población formada y capacitada en el dominio y manejo de las TIC para la vida universitaria; fomentar valores de autenticidad, cultura y felicidad, así como promover una conciencia y unas dinámicas globales sin perder los valores locales. Este elemento se vincula con la necesidad de formar una nueva visión en los actores universitarios, que promueva el gusto por el aprendizaje, la reflexión y el trabajo en equipo mediante redes sinérgicas de vinculación académica. Promueve el aprendizaje continuo e incentiva el desarrollo de proyectos de I+D+I+i (Tkchuck, 2012).

\section{El Concepto Smart UNL}

\section{Resultados}

En la Figura 1 se expone el concepto Smart UNL como una estructura simple y organizada, que, apoyada en tecnologías electrónicas de información y comunicación, conviven para garantizar el correcto desarrollo del quehacer universitario. Este concepto representa la inclusión de información relevante para la correcta y oportuna toma de decisiones, ya sea en aspectos administrativos, académicos, de investigación y vinculación, cuyos datos provienen de distintas fuentes, tanto ordenadas como no ordenadas; este concepto busca crear un entorno favorable para lograr mejoras sustanciales en la convivencia universitaria, haciendo uso intensivo de las tecnologías antes mencionadas. En este contexto, la incorporación de nuevos dispositivos permitirá instituir una integración e interacción de muchos actores al servicio de la institución y en beneficio de su comunidad.

Figura 1

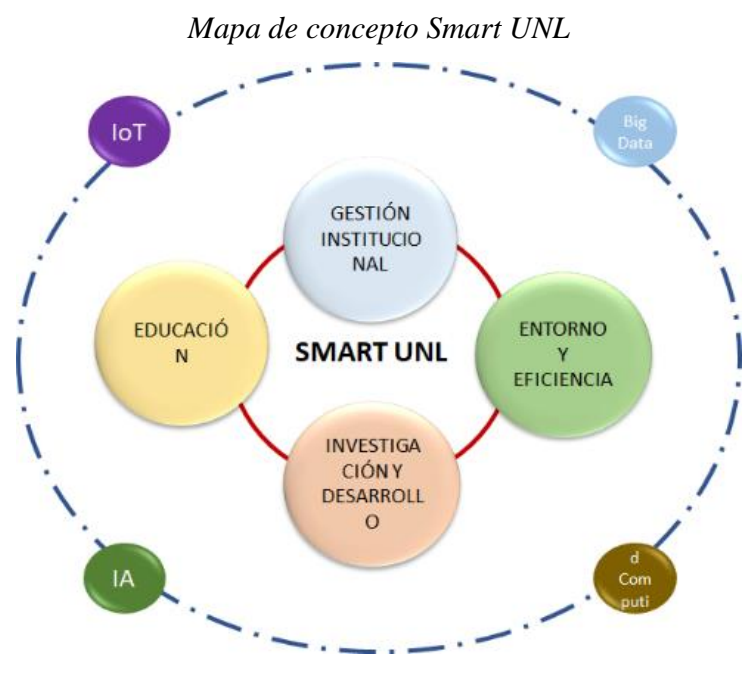




\section{Ejes estratégicos que definen y caracterizan el proyecto Smart UNL}

1) Enlace: este eje estratégico primordial permite integrar en una sola acción las diversas áreas y actores de la institución, con el objetivo de establecer espacios únicos de sinergia que permitan llevar una gestión eficiente a todas las funciones sustantivas de la universidad: educación inclusiva, innovadora y de calidad, investigación científica interdisciplinaria y pertinente, y, finalmente, vinculación social que promueva iniciativas en equipo desde un enfoque colaborativo para la producción de conocimiento y desarrollo comunitario.

2) Empleo: este eje estratégico establece la capacidad de brindar a los estudiantes una formación acorde a la realidad y necesidad laboral del entorno regional y nacional; con este objetivo, se proporciona al alumnado un perfil de formación y egreso emprendedor, creativo y ético.

3) Transferencia: este eje estratégico apunta a la expansión, difusión y compartición de las buenas prácticas institucionales; además, cabe señalar que este eje considera a la innovación tecnológica, los nuevos conocimientos y las estrategias universitarias como insumos importantes dentro de la sociedad digital para contribuir a la resolución de problemáticas diversas, estrechando vínculos con otras instituciones, universidades y empresas.

\section{Ejes de Gobierno de la UNL para espacios Inteligentes}

En la Universidad Nacional de Loja (UNL) se están efectuando cambios en el modelo de gobierno del sistema educativo para el fortalecimiento institucional. Para ello, la mayoría de sus esfuerzos se han encaminado a la capacitación y actualización de su talento humano, sobre todo, haciendo énfasis en la eficacia y eficiencia de la administración y gestión universitaria. De esta forma, la UNL ha propuesto la creación de instrumentos normativos, de planificación y de gestión institucional, guiados por el Plan Estratégico de Desarrollo Institucional-PEDI 2019-2023. Dentro del Plan, la UNL incorpora procesos de autoevaluación y acreditación institucional como elementos clave para identificar las debilidades y fortalezas de los procesos educativos y de gestión. Esta información permitirá edificar una nueva universidad que esté fundamentada en una adecuada gestión académico-administrativa, ágil y que incorpore tecnologías adecuadas que le permitan cumplir con su visión. Ver Figura 2.

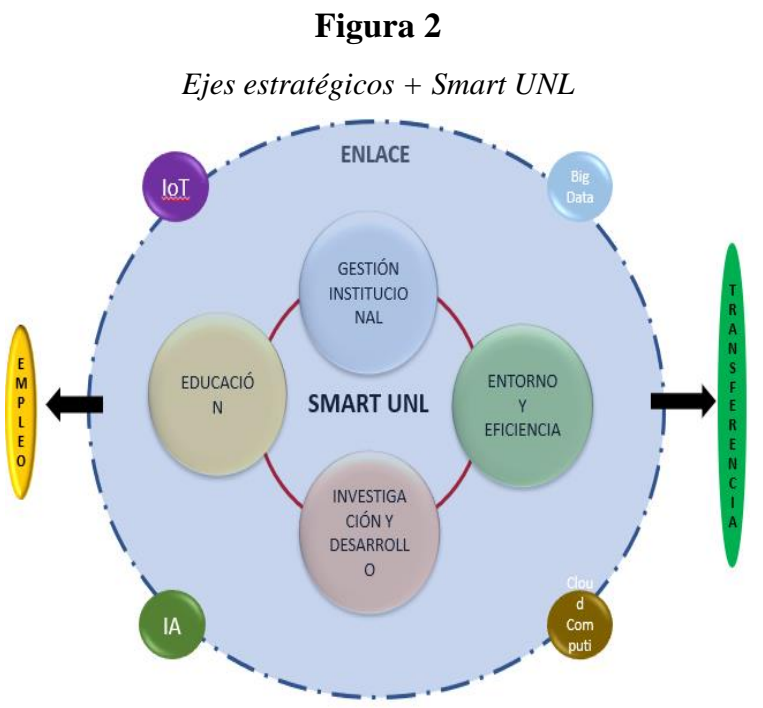

En este sentido, la UNL apuesta por una concepción político-ideológica propia, pero alineada con el modelo de desarrollo del Ecuador, los postulados de convivencia mundial planteados por la Agenda 2030 y los 17 Objetivos de Desarrollo Sostenible (ODS).

Sobre la concepción política-ideológica, la universidad se asienta en tres pilares pensados para la sostenibilidad institucional: i) Marco orientador institucional; ii) Capacidad institucional, y iii) Credibilidad institucional.

Dentro del marco orientador, la concepción político-ideológica de la UNL busca: 
i) La excelencia académica con visión de futuro que se mantenga en sintonía con la evolución de la sociedad y la educación, adaptándose a la actual sociedad del conocimiento con sistemas educativos actualizados, TIC y redes para la libre circulación del conocimiento, reduciendo con ello la brecha cognitiva entre educación y desarrollo; ii) la investigación básica y aplicada con pertinencia para la solución de los problemas reales de la Región Sur del Ecuador y el aporte a la ciencia en la esfera nacional y global; y, iii), la vinculación con la sociedad con responsabilidad social para contribuir desde el accionar de la academia al mejoramiento de las condiciones y calidad de vida de las poblaciones más vulnerables.

En el marco de esta visión, la UNL solicita a todas las instancias universitarias de gestión académica y administrativa integrar en sus competencias, roles y funciones las estrategias, los indicadores y las acciones inclusivas que faciliten mejorar los procesos académicos, de investigación, de vinculación y de gestión administrativa. La Universidad está comprometida en desarrollar un nuevo modelo de gestión pública efectiva para lograr gobernabilidad y gobernanza, de tal manera que esto posibilite disminuir la brecha entre educación y desarrollo.

Dentro de sus políticas, la Universidad apunta a incrementar la investigación científica y, hacia afuera, contribuir a mejorar la capacidad tecnológica de los sectores industriales, así como apoyar en el desarrollo de nuevas tecnologías, investigación e innovación, cumpliendo de este modo con el objetivo 9 de la ODS. Asimismo, se apunta a cumplir con el objetivo 17 de la ODS, mediante el mejoramiento de la cooperación regional e internacional en materia de ciencia, tecnología e innovación, y el acceso a estas.

\section{Ejes tecnológicos de la UNL para espacios inteligentes}

La imparable revolución IoT (Internet of Things) posibilita que cualquier dispositivo se conecte a través de la web: cámaras, sensores, detectores de movimiento, etc., lo que, a su vez, genera grandes volúmenes de datos que deben ser captados, estructurados y almacenados. Por ejemplo, los proyectos de investigación producen una enorme cantidad de información que deberán ser procesadas para convertirse en conocimiento útil en beneficio de la universidad y la sociedad en general. En este sentido, evidenciar el contexto tecnológico actual de la UNL es muy importante, ya que esto permitirá conocer las necesidades tecnológicas que requiere para convertirse en un Smart University; del mismo modo, permitirá identificar y establecer las acciones técnicas que promuevan el establecimiento de sistemas inteligentes con la finalidad de contar con una adecuada gestión administrativa, académica e investigativa. A través del proyecto de investigación Smart UNL, se ha logrado establecer una propuesta de arquitectura "Smart University" (Figura 3), encaminada a almacenar todos los datos generados procedentes de proyectos de investigación, actividades de gestión, acciones académicas, información bibliotecaria y de laboratorios, áreas IoT, etc. Una vez almacenada la información, esta también se podrá procesar, evaluar, visualizar y aplicar, dentro y fuera de la comunidad universitaria, para la ejecución de consultas, estudios, reportes, proyectos, patentes, etc. La arquitectura consta de tres capas fundamentalmente, que, para su interacción, se deben integrar a los tres ejes tecnológicos que la institución necesita para crear espacios inteligentes.

Figura 3

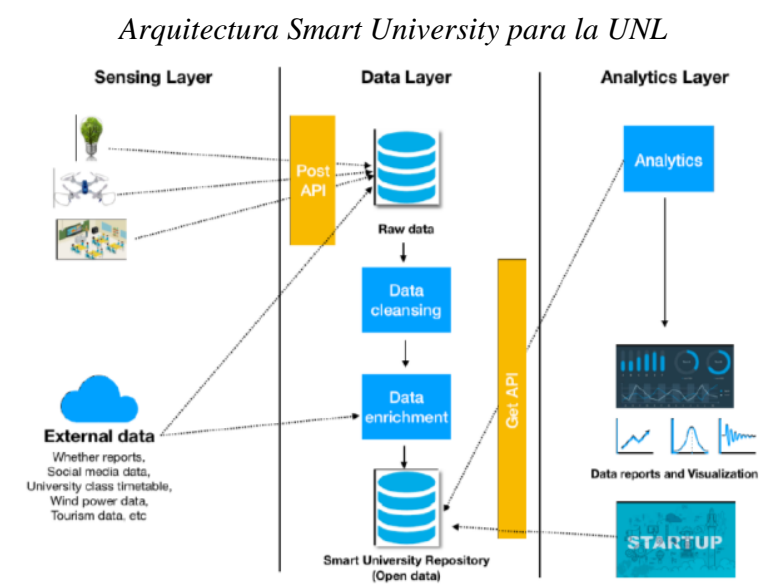


La relación entre la arquitectura propuesta y los ejes tecnológicos se fundamenta en los elementos siguientes:

1) Telecomunicaciones y Sensing Layer: la infraestructura de telecomunicaciones que permite brindar conectividad dentro y fuera de los predios universitarios se convierte en la vía de transporte e interacción de toda la información generada en la capa de extracción de datos (Sensing Layer), según la Arquitectura Smart University (Figura 3); la tecnología requerida transportaría ágilmente información generada por sensores, redes sociales, páginas web, datos que se hayan capturado manualmente y, luego, digitalizado en encuestas, entrevistas, registros físicos, etc.; esto aportaría con una gran cantidad de información para diferentes análisis y posteriores decisiones, lo que otorgaría mayor control y transparencia a la infraestructura de comunicación universitaria, facilitando de este modo el establecimiento de estrategias que garanticen la eficiencia y la sostenibilidad de cada facultad de la UNL, ya sean en los ámbitos de la movilidad, la energía, la gobernanza, la academia, la investigación o la unión de todas ellas. Como se manifestó en apartados anteriores, invariablemente, las TIC ocupan un lugar transversal en cualquiera de los escenarios que aspiran a convertirse en espacios inteligentes dentro de la universidad.

2) Bases de datos (Data Layer): la necesidad de infraestructura de almacenamiento de información es primordial. Para ello, se requiere establecer los espacios para ubicar los servidores de bases de datos y adicionales. En la capa Data Layer se deberán almacenar todos los datos capturados de la capa Sensing Layer, específicamente, en un servidor de datos crudos; luego de ser almacenados estos pasan por un proceso de limpieza y, luego, por un proceso de enriquecimiento, si es necesario, para, finalmente, ser almacenados en otro servidor centralizado y global donde estará toda la información procesada. La información en este servidor operará con la filosofía Open Data.

3) Software y herramientas Informáticas (Analytics Layer): este eje permitirá a la institución desarrollar los mecanismos informáticos más adecuados para la correcta interacción con la información proveniente de los diferentes puntos generadores de datos. Aunque, necesariamente, el software y las herramientas informáticas están asociadas a las capas anteriores, para el caso de la Analytics Layer, también estas permitirán la obtención de datos procesados del repositorio central para generar análisis, reportes, visualizaciones, animaciones, aplicaciones, etc. Al tener la información centralizada y disponible para toda la comunidad universitaria, los estudiantes, los profesores e, incluso, los aliados estratégicos podrán usar los datos procesados para generar nuevas ideas de negocios (startups, Networking, etc.); asimismo, los investigadores podrán generar mayor conocimiento (connections) y los administrativos podrán tener fácil y dinámico acceso a la información y a los sistemas institucionales.

La Figura 4 muestra el espectro de acción donde los ejes estratégicos de gobierno y tecnológicos, sustentados en una arquitectura Smart, aportan, interactúan y operan a través de una maniobra mancomunada para crear y brindar un entorno inteligente a la universidad.

Figura 4

Resultado de transversalidad de Ejes estratégicos, de gobierno y tecnológicos para Smart UNL

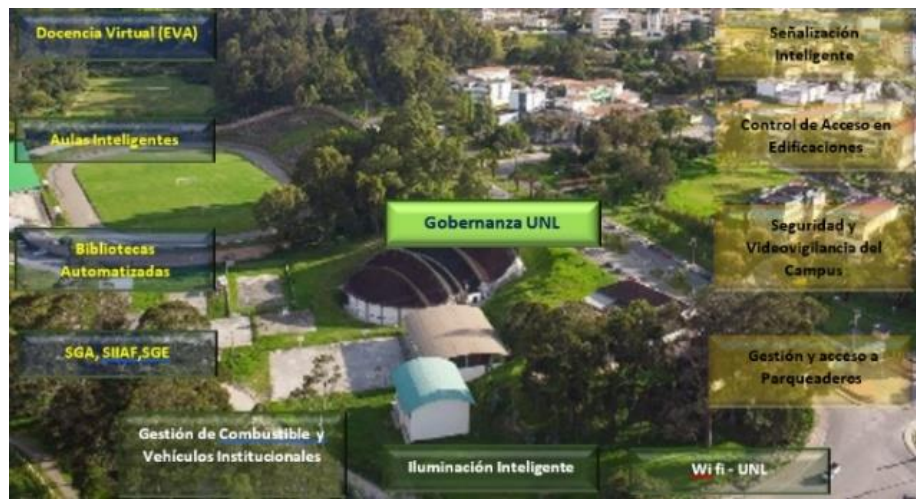

Finalmente, en la Figura 5 se consolida la visión de todos los recursos expuestos en el documento, los mismos que, ya instaurados, permitirán el despliegue del concepto Smart UNL. 
Figura 5

Operatividad de Ejes Estratégicos + Smart UNL + Arquitectura

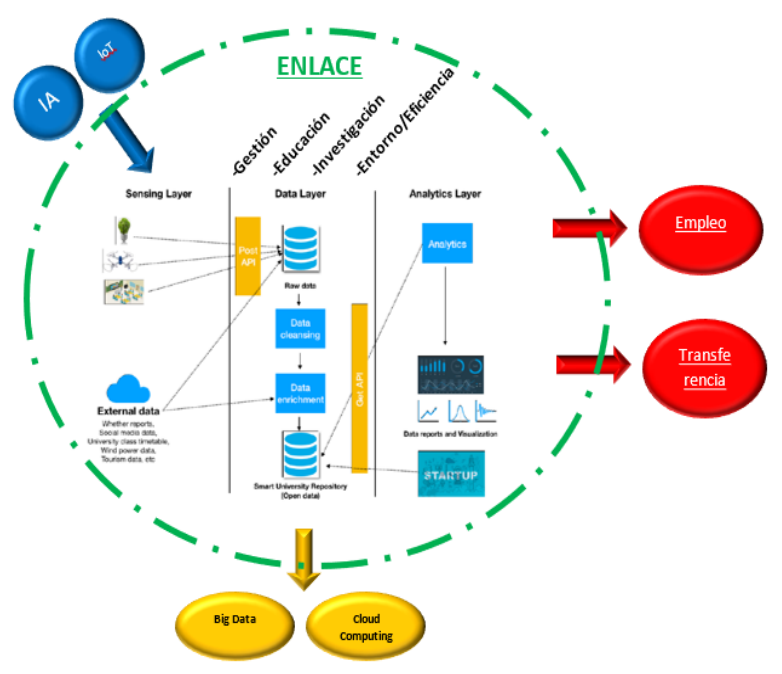

\section{Conclusiones}

En la actualidad, alrededor del mundo, en las instituciones universitarias son escasos los proyectos vinculados al concepto Smart University, aunque los que se han planteado muestran un creciente posicionamiento como alternativa de excelencia para la gestión universitaria.

En el Ecuador, el concepto de universidad inteligente aún se muestra como un reto a ser asumido por las instituciones de educación superior; poco o nada se ha avanzado en esta línea; únicamente, la Escuela Politécnica del Litoral (ESPOL) ha dado algunos pasos al respecto. Por ejemplo, el trabajo de investigación Operational framework proposal for ESPOL university 2.0 smart campus implementation presenta una propuesta para transitar hacia futuros escenarios, integrando soluciones de TI, eficiencia, seguimiento y uso óptimo de recursos, departamentos de energía, etc.; todos definidos como divisiones abiertas que podrían conducir al campus politécnico a convertirse en una ciudad autónoma.

La Universidad Nacional de Loja, a través del Proyecto Smart UNL, pretende introducir en la institución los conceptos de universidad inteligente, y, para ello, como primer paso, planea aplicar el concepto de eficiencia energética en el área eléctrica de la universidad, que se traduce en la implementación de tecnología de iluminación inteligente y eficiente, además, en el establecimiento de acciones concretas para reducir el consumo energético de sus edificaciones, aportando así a la economía de la universidad y a la conservación del medio ambiente.

La evolución tecnológica hace que sea prácticamente imposible que las instituciones de educación superior no actúen en función de este desarrollo; sus prácticas académicas, de vinculación e investigación deberán avanzar junto con la tecnología.

La UNL, como principal institución pública en la Región 7 del Ecuador, requiere y precisa actualizar sus prácticas de gestión, administración y desarrollo basado en tecnologías para mantener un ritmo competitivo en la investigación y vinculación frente a otras entidades educativas.

La propuesta de arquitectura para gestión de la información, utilizando datos abiertos garantizados por el proyecto Smart UNL, puede significar el camino de la UNL hacia una universidad inteligente.

La institución cuenta con una red de comunicaciones actualizada; su infraestructura tecnológica de software y hardware es aplicable o adaptable a consideraciones de Smart University, por lo que la aplicación del concepto de Smart campus es viable para la UNL. 
La innovación y transformación digital que trae consigo la tecnología 4.0 requiere un cambio en el ecosistema de las TIC que integre dispositivos móviles, tabletas o teléfonos inteligentes a las redes inalámbricas de alta velocidad y alto consumo de aplicaciones móviles. Esto permitirá transformar el entorno del usuario universitario hacia nuevas posibilidades que fortalezcan su formación profesional.

\section{Referencias}

P. Pizarro. (2015). La evolución de la educación en Latinoamérica. Identidad 21, Revista Digital Universidad Siglo 21. https://identidad.21.edu.ar/la-evolucion-de-la-educacion-en-latinoamerica/

C. Tkchuck. (2015) La Universidad Inteligente: un nuevo paradigma de gestión institucional. Argentina: Universidad Nacional de Quilmes.

http://libros.uvq.edu.ar/assets/libro3/la_universidad_inteligente_un_nuevo_paradigma_de_gestin_instit ucional.html

M. Viñán, L. Jacome, L. Rodríguez, A.Vega, C. Campoverde. (2020). Smart University: An architecture proposal for information management using Open Data for research projects. Colombia: International Conference on Information Technology \& Systems ICITS, 172-178.

UTI. (2020). Condiciones Tecnológicas y Conectividad a Internet, UTI., Loja-Ecuadro: Universidad Nacional de Loja,

J. Sanchez, F. Macia, J. Berna, I. Lorenzo, A. Fuster. (2016). Smart University: hacia una universidad más abierta. Marcombo-España:

V. Uskov, J. Bakken, R. Howlett, L. Jain. (2018). Smart Universities: Concepts, Systems, and Technologies. USA: Springer.

M. Zapata. (2018). La universidad inteligente Archivo de la etiqueta: Smart universities. https://red.hypotheses.org/tag/smart-universities

M. Caţă. (2015). Smart university, a new concept in the Internet of Things. IEEE 14th RoEduNet International Conference - Networking in Education and Research (RoEduNet NER), Romania.

M. Jamrisko, W. Lu. (2020). Germany Breaks Korea's Six-Year Streak as Most Innovative Nation. https://skylineimmigration.com/germany-breaks-koreas-six-year-streak-innovative-nation/

Unesco. (1998). Declaración Mundial sobre la Educación Superior en el siglo XXI. IESALAC. (1989). Visión y Acción. Educación Superior y Sociedad, 9(2).

https://www.iesalc.unesco.org/ess/index.php/ess3/issue/view/21

M. García, J. Reyes y G. Godínez. (2017). Las Tic en la educación superior, innovaciones y retos”. Revista Iberoamericana de las Ciencias Sociales y Humanísticas: RICSH, (612), 299-316.

Sangrá, Enseñar y aprender en la virtualidad”'. Educar, (28), 117-131. Disponible en: https://raco.cat/index.php/Educar/article/view/20746/20586

Educación Superior, Ciencia, Tecnología, Innovación y Saberes Ancestrales. (2018). Educación Superior, Ciencia, Tecnología, Innovación y Saberes Ancestrales en Cifras.

https://www.educacionsuperior.gob.ec/wpcontent/uploads/downloads/2019/01/00_Nacional_Educacio n_Superior_en_Cifras_Diciembre_2018.pdf

M. Ferreyra, C.Avitabile, J. Botero, F Haimovich, S.Urzúa. (2017). La educación superior en América Latina y el Caribe. Washington: Banco Mundial.

https://openknowledge.worldbank.org/bitstream/handle/10986/26489/211014ovSP.pdf?sequence=5\&is Allowed=y

Grupo Especial sobre Educación Superior y Sociedad. (2000). La Educación Superior en los países en desarrollo: Peligros y Promesas. Banco Mundial: Wachintg. 
https://documents1.worldbank.org/curated/en/630041467998505995/pdf/2018210educaci1paises0en0d esarrollo.pdf

Banco Mundial. (2017). La educación superior se expande en América Latina y el Caribe, pero aún no desarrolla todo su potencial. https://www.bancomundial.org/es/news/press-release/2017/05/17/highereducation-expanding-in-latin-america-and-the-caribbean-but-falling-short-of-potential

Centro de Estudios de la Educación Argentina (2021). Este 2021 será crítico para la Educación. http://repositorio.ub.edu.ar/bitstream/handle/123456789/9159/CEA_febrero-marzo_2021.pdf 